\title{
ASSESSMENT OF THE RELEVANCE OF INFORMATION DERIVED FROM THE UNMIXING OF POLARIMETRIC RADAR IMAGES
}

\author{
Sébastien Giordano ${ }^{1}$, Grégoire Mercier ${ }^{2}$, Jean-Paul Rudant ${ }^{1}$ \\ 1. IGN-MATIS Lab., Université Paris Est, France \\ 2 : Institut Mines-Telecom / Telecom Bretagne, CNRS UMR 6285 Lab-STICC, team CID, France
}

\begin{abstract}
A new method to unmix radar polarimetric images with optical images was proposed. This method has pointed out that the unmixing model is able to split off polarimetric information on a land cover type basis. In this paper unmixed radar polarimetric images obtained are compared with the observed ones in non-mixed conditions. Then, Cloude and Pottier decomposition is performed on the unmixed and observed radar images to asses whether the understanding of physical scattering mechanisms is improved with the unmixing. Finally, a classification experiment is designed to determine whether this fusion framework make the transfer of information from the optical images to the unmixed radar images possible.
\end{abstract}

Index Terms - unmixing, radar polarimetry, polarimetric decomposition.

\section{INTRODUCTION}

Data fusion can traditionally be performed at three different levels : the data level (observations), the intermediate level (features) and finally the decision level (classifications). In particular, at the data level, combining observations from optical images and polarimetric radar images is challenging as these observations are of very different types. Polarimetric radar decompositions [1] aim at retrieving physical scattering properties of the objects (such as surface, double bounce or volume scattering), or some statistical properties (entropy, anisotropy and alpha angle in the case of Cloude and Pottier decomposition). If different land cover types are mixed together in the cell resolution the polarimetric parameters obtained might not well describe any of these land cover types. The main idea of the unmixing method proposed is to split off polarimetric information on a land cover basis before applying usual polarimetric decompositions.

This work was partially supported thanks to a support from the European Union, the Brittany Region and the Brest Metropole Oceane to the VIGISAT project, in the framework of a FEDER grant Présage \# 32635 and a partnership with CLS for providing the data, under the contracts\# 08 GET 13M and 09 GET 11M.

\section{METHOD}

The unmixing equations used for two land cover types are detailed in [2]. The equations derive from an extent of Goodman hypotheses [3] for the case of two different land cover types 0 and 1 . The optical-derived parameter $\mu \in[0,1]$ characterising the proportion of type 0 and 1 was introduced. Within these hypotheses it was found that the linear form in equation 1 can be used to unmix the observed radar covariance matrix. As a result, the unmixed covariance matrices $C_{0}$ and $C_{1}$ could be estimated. This algorithm was performed on a study area in Provence (South-East of France) considering two land cover classes : Forested area (0) and Bare soil (1). The data used in this experiment are $25 \mathrm{~cm}$ resolution aerial images acquired on June 22, 2012 in addition with a Radarsat-2 full polar image obtained on June 27, 2012 (pixel size in slant range $4,73 m \times 4,76 m)$.

$$
[C]=\mu\left[C_{0}\right]+(1-\mu)\left[C_{1}\right]
$$

As the objective is to assess whether thematic information can be retrieved from the unmixing, the workflow detailed in Figure 1 was applied to the observed $C$ and the unmixed $C_{0}$ and $C_{1}$ covariance matrices.

\section{COVARIANCE MATRICES}

Averaged $C_{11}, C_{13}, C_{22}$ and $C_{33}$ elements were calculated for 50 intervals of $\mu$. Plots (b), (d), (f) and (h) in Figure 2 show that on the study area observed $C_{11}, C_{13}, C_{22}$ and $C_{33}$ increase with $\mu$. The non-mixed conditions of the observed $C$ matrix are obtained when $\mu \rightarrow 0$ for bare soil and $\mu \rightarrow 1$ for forest. Observed $C$ matrix calculated in non-mixed conditions seem to be relevant with the elements estimated with the unmixing. For example, $C_{22}$ element goes from $0.3 \times 10^{6}$ d.n. to $0.5 \times 10^{6}$ d.n., which order of magnitude is consistent with the unmixed $C_{0_{22}}$ (forest) and $C_{1_{22}}$ (bare soil).

\section{CLOUDE AND POTTIER DECOMPOSITION}

In order to evaluate whether the unmixed product can produce more reliable results for large scale land cover classification, 

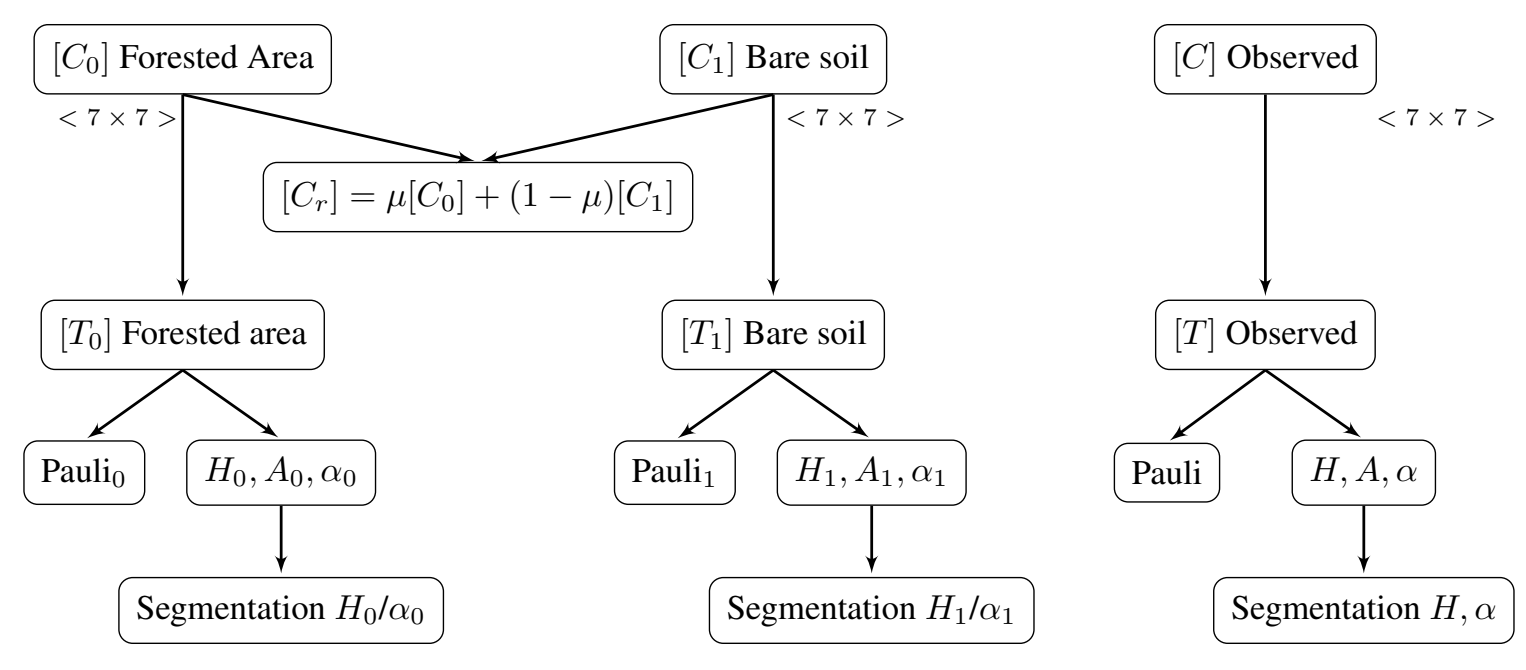

Fig. 1: Polarimetric Radar Decompositions

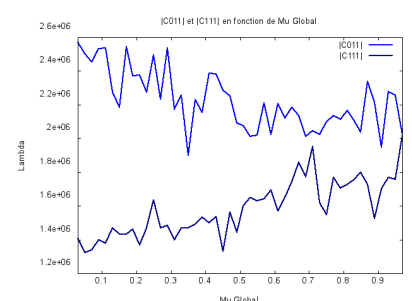

(a) $C_{0_{11}}, C_{1_{11}}$

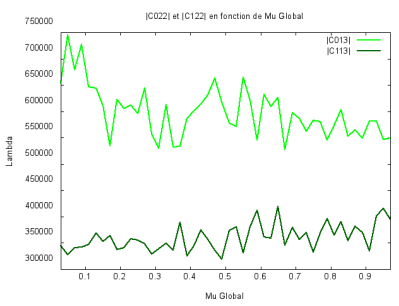

(e) $C_{0_{22}}, C_{1_{22}}$

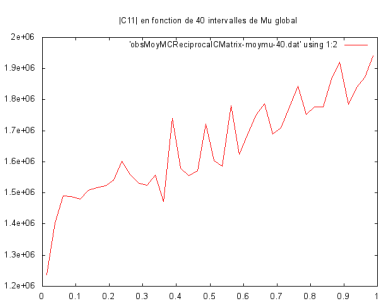

(b) $\mathrm{C} 11$

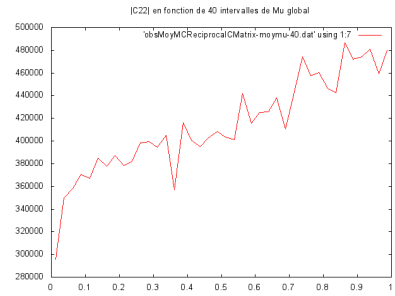

(f) $\mathrm{C} 22$

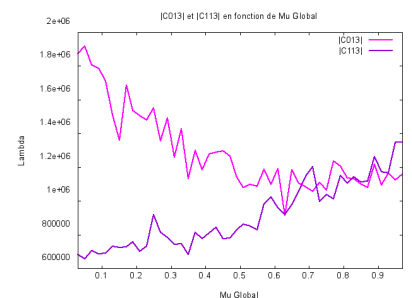

(c) $C_{0_{13}}, C_{1_{11}}$

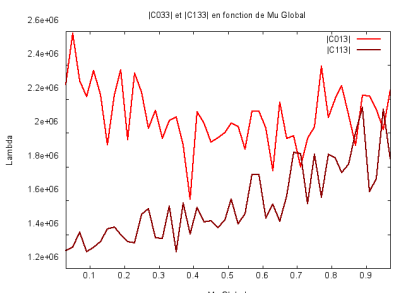

(g) $C_{0_{33}}, C_{1_{33}}$

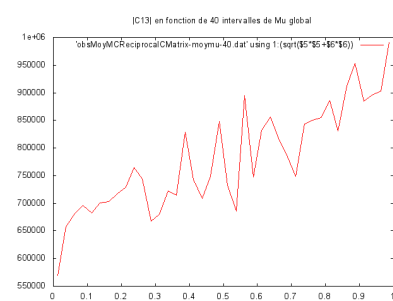

(d) $\mathrm{C} 13$

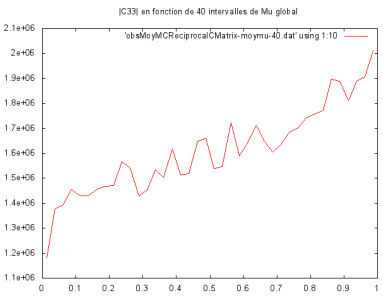

(h) C33

Fig. 2: Elements of observed $C$ and unmixed $C_{0}$ and $C_{1}$ depending on $\mu$

the $(H, \alpha, A)$ parameters of the Cloude and Pottier decomposition [4] were computed from the the observed covariance matrix $C$ and the unmixed $C_{0}$ and $C_{1}$ covariance matrices as described in Figure 1. Figure 3 shows the physical scattering characteristics defined by [4] obtained by segmenting the $(H, \alpha)$ plane.

In addition with the $\mathrm{Z} 6, \mathrm{Z5}$ and $\mathrm{Z2}$ behaviours that occur in the observed $(H / \alpha)$ segmentation, $\left(H_{0} / \alpha_{0}\right)$ segmentation introduces new types of scattering mechanisms. Z4 scattering type traducing dihedral scattering with moderate entropy (forest double-bounce scattering) appears in the forest when the canopy cover is low. Isolated trees are characterised by $Z 7$ (isolated dihedral scatterers with low entropy) and Z8 (isolated dipole scatterer from vegetation) segmentation types.

\section{CLASSIFICATION EXPERIMENT}

Field work on the study area lead to a 5 classes land cover nomenclature : forested area, bare calcareous soil, thyme grassland, annual grass and multiannual grass. An objectbased classification was performed on the multi-spectral optical images considering these 5 thematic classes using the $\mu$ parameter (optical information) and Cloude and Pottier parameters from the observed radar images $(H, A, \alpha)$ and the unmixed images $\left(H_{0}, A_{0}, \alpha_{0}\right)$ and $\left(H_{1}, A_{1}, \alpha_{1}\right)$.

On this study area the 5 land cover types can be retrieved with the optical images, as a result ground truth data was produced with a classification of the optical images. Random forests importance of variables ([5]) was performed on the 


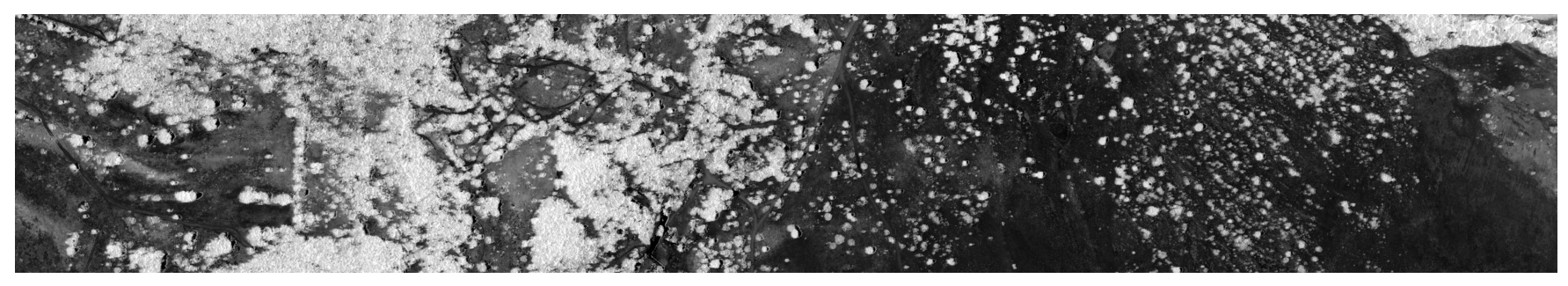

(a) optical NDVI of the study area

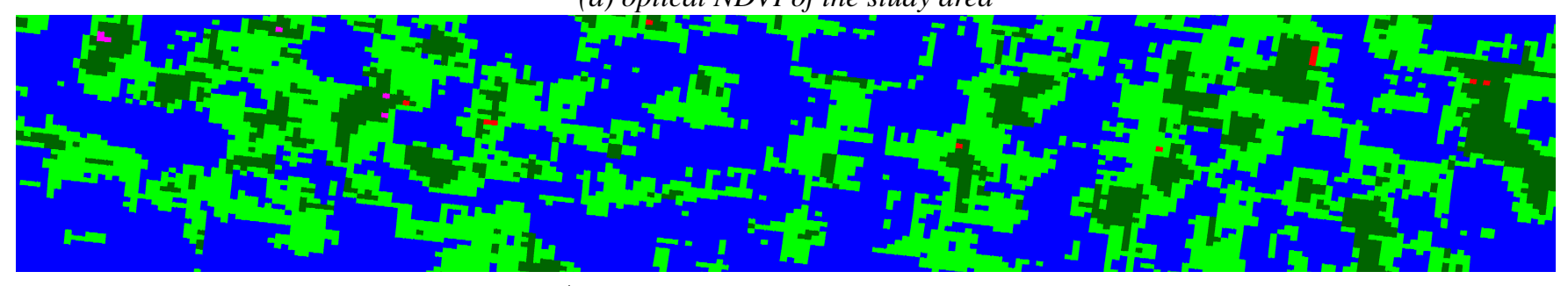

(b) $H / \alpha$ segmentation types from the observed $T$

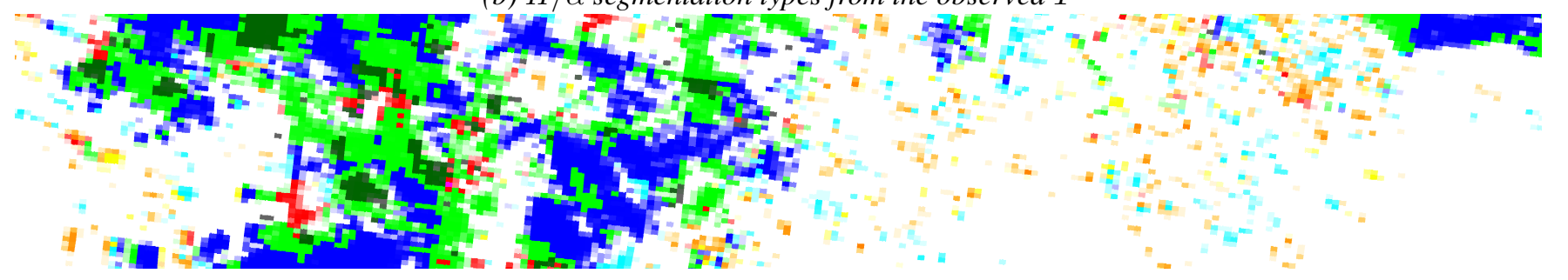

(c) $H_{0} / \alpha_{0}$ segmentation types from $T_{0}$ (forest)

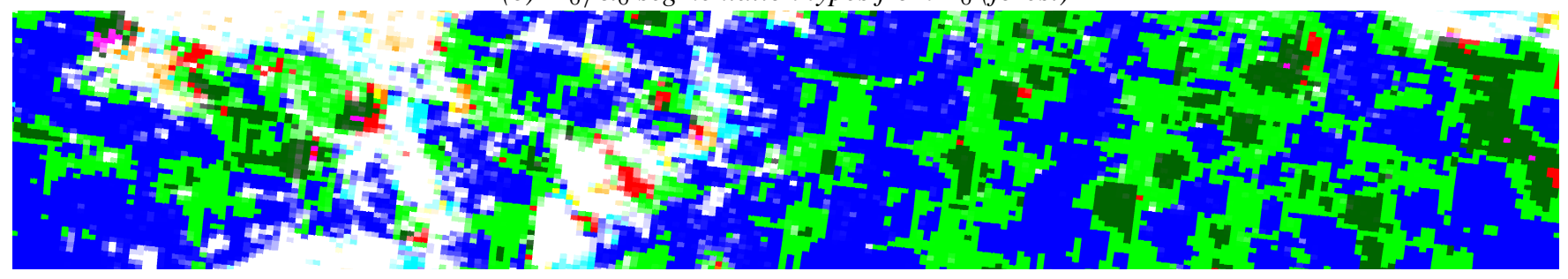

(d) $\left(H_{1} / \alpha_{1}\right)$ segmentation types from $T_{1}$ (bare soil)

\begin{tabular}{|l|l|l|l|l|l|l|l|l|}
$\mathbf{Z 1}$ & $\mathbf{Z 2}$ & $\mathbf{Z 3}$ & $\mathbf{Z 4}$ & $\mathbf{Z 5}$ & $\mathbf{Z 6}$ & $\mathbf{Z 7}$ & 78 & $\mathbf{2 9}$ \\
\hline
\end{tabular}

Fig. 3: Segmentation $\mathrm{H} / \alpha$ from $T, T_{0}$ and $T_{1}$, colour saturation : $\mu$

datasat. Results are given in Table 1.

Then, a classification using the Random Forest classifier ([5]) was performed and evaluated through cross-validation (automatic split of 3856 samples with $40 \%$ train, $60 \%$ control). Table 3 shows overall precision, recall and F-measure of the classification for 6 combination of parameters defined in Table 2 : optical information (configuration $\mathbf{A}$ ), observed radar (configuration $\mathbf{B}$ ), unmixed radar (configuration $\mathbf{C}$ ), optical information and observed radar (configuration D), optical information and unmixed radar (configuration E), optical information, observed and unmixed radar (configuration $\mathbf{F}$ ).

On the one hand the optical-derived parameter $\mu$ is the highest ranked parameter. It provides the major part of the information and leads in configuration $\mathrm{A}$ to 0.639 for Fmeasure.
Table 1: Radar Parameters Importance

\begin{tabular}{ccc}
\hline Ranking & Attribute & Importance \\
\hline 1 & $\mu$ & 0.293 \\
2 & $H_{0}$ & 0.165 \\
3 & $H_{1}$ & 0.110 \\
4 & $A_{0}$ & 0.086 \\
5 & $A_{1}$ & 0.078 \\
6 & $\alpha_{0}$ & 0.0560 \\
7 & $\alpha$ & 0.054 \\
8 & $\alpha_{1}$ & 0.054 \\
9 & $H$ & 0.052 \\
10 & $A$ & 0.051 \\
\hline
\end{tabular}


Table 2: Configuration

\begin{tabular}{cc}
\hline Config. & Composition \\
\hline $\mathbf{A}$ & $\mu$ \\
\hline $\mathbf{B}$ & $(H, \alpha, A)$ \\
\hline $\mathbf{C}$ & $\left(H_{0}, \alpha_{0}, A_{0}\right) \cup\left(H_{1}, \alpha_{1}, A_{1}\right)$ \\
\hline $\mathbf{D}$ & $\mu \cup(H, \alpha, A)$ \\
\hline $\mathbf{E}$ & $\mu \cup\left(H_{0}, \alpha_{0}, A_{0}\right) \cup\left(H_{1}, \alpha_{1}, A_{1}\right)$ \\
\hline $\mathbf{F}$ & $\mu \cup(H, \alpha, A) \cup\left(H_{0}, \alpha_{0}, A_{0}\right) \cup\left(H_{1}, \alpha_{1}, A_{1}\right)$ \\
\hline
\end{tabular}

Table 3: Evaluation

\begin{tabular}{cccc}
\hline Configuration & Precision & Recall & F-Measure \\
\hline A & 0.615 & 0.666 & 0.639 \\
\hline B & 0.348 & 0.379 & 0.363 \\
\hline C & 0.593 & 0.664 & 0.626 \\
\hline D & 0.665 & 0.729 & 0.696 \\
\hline E & 0.684 & 0.735 & 0.709 \\
\hline F & 0.683 & 0.738 & 0.709 \\
\hline
\end{tabular}

On the other hand observed $(H, \alpha, A)$ parameters (configuration $\mathrm{B}$ ) lead to poorer classification precision whereas F-measure of unmixed radar (configuration $\mathrm{C}$ ) is close to the configuration A F-measure. The first conclusion that can be drawn is that information from optical images seems to be correctly transferred in the fusion process. Moreover, $\left(H_{0}, \alpha_{0}, A_{0}\right)$ and $\left(H_{1}, \alpha_{1}, A_{1}\right)$ are more significant than $(H, \alpha, A)$ for classification (Table 1$)$.

Combination of radar and optical images produces the best classification results either $\mu$ parameter is combined with the observed radar or the unmixed radar parameters.

As a result we cannot conclude that greater information has been produced with the unmixing. Indeed ground truth was done using optical images and the optical-derived parameter $\mu$ is by itself discriminating. An another experiment has to been designed. For instance, we hope to prove that the unimixing algorithm can improve vegetation volume or canopy cover estimation with radar polarimetry. In that particular case, ground truth can be done using aerial LiDAR data (available on this area) and optical images can only be used to distinguish between soil and forest land cover types.

\section{REFERENCES}

[1] S. R. Cloude and E. Pottier, "A review of target decomposition theorems in radar polarimetry," IEEE Transactions on Geoscience and Remote Sensing, vol. 34, no. 2, pp. 498-518, Mar. 1996.

[2] S. Giordano, G. Mercier, and J.-P. Rudant, "Unmixing polarimetric radar images based on land cover type before target decomposition," in IGARSS, 2014, pp. 2790-2793.

[3] J. W. Goodman, "Statistical properties of laser speckle patterns," Laser speckle and related phenomena, vol. 9, pp. 9-75, 1975.

[4] S. R. Cloude and E. Pottier, "An entropy based classification scheme for land applications of polarimetric SAR.," IEEE Transactions on Geoscience and Remote Sensing, vol. 35, no. 1, pp. 68-78, 1997.

[5] L. Breiman, "Random forests," Machine Learning, vol. 45, no. 1, pp. 5-32, 2001. 